\title{
Removal of Chromophore-Proximal Polar Atoms Decreases Water Content and Increases Fluorescence in a Near Infrared Phytofluor
}

\section{OPEN ACCESS}

Edited by:

Tilo Mathes,

Vrije Universiteit Amsterdam,

Netherlands

Reviewed by:

Petr Leiman,

Ecole Polytechnique Federale de

Lausanne, Switzerland

Wolfgang Gärtner,

Max-Planck-Institut für Chemische

Energiekonversion, Germany

Hernán Ruy Bonomi,

Fundación Instituto Leloir-CONICET,

Argentina

*Correspondence:

Heli Lehtivuori

heli.lehtivuori@jyu.fi;

Katrina T. Forest

forest@bact.wisc.edu

Specialty section:

This article was submitted to

Biophysics,

a section of the journal

Frontiers in Molecular Biosciences

Received: 01 August 2015 Accepted: 02 November 2015

Published: 25 November 2015

Citation:

Lehtivuori H, Bhattacharya S, Angenent-Mari NM, Satyshur KA and

Forest KT (2015) Removal of Chromophore-Proximal Polar Atoms

Decreases Water Content and Increases Fluorescence in a Near Infrared Phytofluor.

Front. Mol. Biosci. 2:65.

doi: 10.3389/fmolb.2015.00065

\author{
Heli Lehtivuori ${ }^{1,2 *}$, Shyamosree Bhattacharya ${ }^{1}$, Nicolaas M. Angenent-Mari ${ }^{1}$, \\ Kenneth A. Satyshur ${ }^{1}$ and Katrina T. Forest ${ }^{1 *}$ \\ ${ }^{1}$ Department of Bacteriology, University of Wisconsin-Madison, Madison, WI, USA, ${ }^{2}$ Department of Physics, Nanoscience \\ Center, University of Jyväskylä, Jyväskylä, Finland
}

Genetically encoded fluorescent markers have revolutionized cell and molecular biology due to their biological compatibility, controllable spatiotemporal expression, and photostability. To achieve in vivo imaging in whole animals, longer excitation wavelength probes are needed due to the superior ability of near infrared light to penetrate tissues unimpeded by absorbance from biomolecules or autofluorescence of water. Derived from near infrared-absorbing bacteriophytochromes, phytofluors are engineered to fluoresce in this region of the electromagnetic spectrum, although high quantum yield remains an elusive goal. An invariant aspartate residue is of utmost importance for photoconversion in native phytochromes, presumably due to the proximity of its backbone carbonyl to the pyrrole ring nitrogens of the biliverdin (BV) chromophore as well as the size and charge of the side chain. We hypothesized that the polar interaction network formed by the charged side chain may contribute to the decay of the excited state via proton transfer. Thus, we chose to further probe the role of this amino acid by removing all possibility for polar interactions with its carboxylate side chain by incorporating leucine instead. The resultant fluorescent protein, WiPhy2, maintains BV binding, monomeric status, and long maximum excitation wavelength while minimizing undesirable protoporphyrin IX $\alpha$ binding in cells. A crystal structure and time-resolved fluorescence spectroscopy reveal that water near the BV chromophore is excluded and thus validate our hypothesis that removal of polar interactions leads to enhanced fluorescence by increasing the lifetime of the excited state. This new phytofluor maintains its fluorescent properties over a broad pH range and does not suffer from photobleaching. WiPhy2 achieves the best compromise to date between high fluorescence quantum yield and long illumination wavelength in this class of fluorescent proteins.

Keywords: chromophore binding domain (CBD), Deinococcus radiodurans, Wisconsin infrared phytofluor (WiPhy2), tetrapyrrole, excitation-emission matrix (EEM) 


\section{INTRODUCTION}

Fluorophores active in the near infrared (NIR) attract ongoing attention due to their diverse applications in biomedical research, materials science and related fields. They allow imaging with minimal autofluorescence and light scattering in animals, and deep tissue penetration (Weissleder, 2001). In vivo, real-time advanced imaging studies and vascular mapping of the heart and brain, the visualization of tumors and plaques, and guided surgery are aspects of fundamental research and translational applications that will benefit substantially from the creation of improved NIR fluorophores. The development of simple, stable, non-toxic, modular, and small molecular weight NIR platforms is thus of great interest to the biomedical community and has proceeded both in the realm of chemical biology and fluorescent proteins. In the former category, there are several classes of small molecule NIR dyes available including nanoparticles, cyanine dyes, phthalocyanine, and squaraine dyes (Escobedo et al., 2010; Hahn et al., 2011; Luo et al., 2011; Gibbs, 2012; Battistelli et al., 2015). The promise of genetically encoded NIR fluorescent proteins has, on the other hand, led to a renaissance in research of engineered fluorescent proteins, based both on Green Fluorescent Protein-like $\beta$-barrel folds and more recently on bacteriophytochromes (Zhang et al., 2002; Gibbs, 2012; Guo et al., 2014; Marx, 2014).

Bacteriophytochromes (BphPs) are promising design templates for NIR fluorescent proteins. Their covalent association with the linear tetrapyrrole biliverdin IX $\alpha$ (BV) allows BphPs to absorb light in the red and far-red region of the spectrum. As an intermediate of normal mammalian heme catabolism, BV does not necessarily need to be provided exogenously in order to achieve in vivo fluorescence. Thus, great effort has gone into improving the photophysical and chemical properties of microbial phytochrome-based dyes in the last decade (Marx, 2014). Fluorescence quantum yields have increased, molecular weight has decreased, and excitation wavelengths are both extended farther to the red (above $700 \mathrm{~nm}$ ) while also being available in multiple colors (Figure 1). The use of these tools promises to extend fluorescence imaging to live animals. Further development in all of these areas will bring phytofluors into quotidian use.

The family of phytochromes shares a conserved photosensory protein core consisting of a PAS (er/Arndt/Sim) domain, a GAF (GMP phospho-diesterase/adenyl cyclase/FhlA) domain and a PHY (phytochrome) domain. While full-length phytochromes are required for biological activity, fluorescence protein development is concentrated to PAS and GAF domains, which together form a chromophore-binding domain (CBD). Wildtype BphPs are dimers, but the strength of the dimerization interface varies among phytochromes (Takala et al., 2015). To increase BphP utility as a fluorophore, residues in this GAF dimer interface have been rationally mutated to create a monomer (Bhattacharya et al., 2014; Yu et al., 2014).

This initial monomeric CBD from Deinococcus radiodurans $\left(\mathrm{DrCBD}_{\text {mon }}\right)$ has a low fluorescence quantum yield $(0.029 \pm$ 0.001; Auldridge et al., 2012; Bhattacharya et al., 2014). In order to rationally improve this yield, one can imagine engineering the protein to affect changes in the kinetics of the competing processes that take place in the excited state, in particular internal conversion or isomerization in the $\mathrm{BV} \mathrm{C15}=\mathrm{C} 16$ double bond leading to the first relatively stable photoproduct (Lumi-R). Much attention has been paid in particular to the Y263F and $\mathrm{D} 207 \mathrm{H}$ substitutions, in large part because of the critical roles these positions play in the normal photocycle (Sineshchekov et al., 2014). Recently it has been shown that the $\mathrm{H} 207$ residue is not required for enhanced fluorescence of Infrared Fluorescent Protein (IFP)1.4 (Shu et al., 2009; Bhattacharya et al., 2014) and does not markedly increase fluorescence in Wisconsin Infrared Phytofluor (WiPhy $=$ DrCBD $_{\text {mon }}-\mathrm{Y} 263 F / D 207 H$ ) (Auldridge et al., 2012). Indeed in IFP2.0, this position is a Thr (Yu et al., 2014). The side chains introduced by these mutations change the hydrogen-bonding network of the binding pocket (Toh et al., 2010, 2011a,b; Zienicke et al., 2011, 2013; Auldridge et al., 2012; Bhattacharya et al., 2014; Yu et al., 2014).

Our motivation in this study has been to explore the effects of a nonpolar substitution of residue 207, which is in closest proximity to the four nitrogen atoms of BV and the ordered pyrrole water found interacting with three of them. We chose to substitute Leu because it is the nonpolar side chain whose structure most closely mimics that of the native Asp. The size of Leu should prevent adventitious binding of Protoporphyrin IX $\alpha$ (PPIX $\alpha)$, which interacts covalently with H207-carrying variants (Fischer and Lagarias, 2004; Wagner et al., 2008; Lehtivuori et al., 2013; Burgie et al., 2014). The

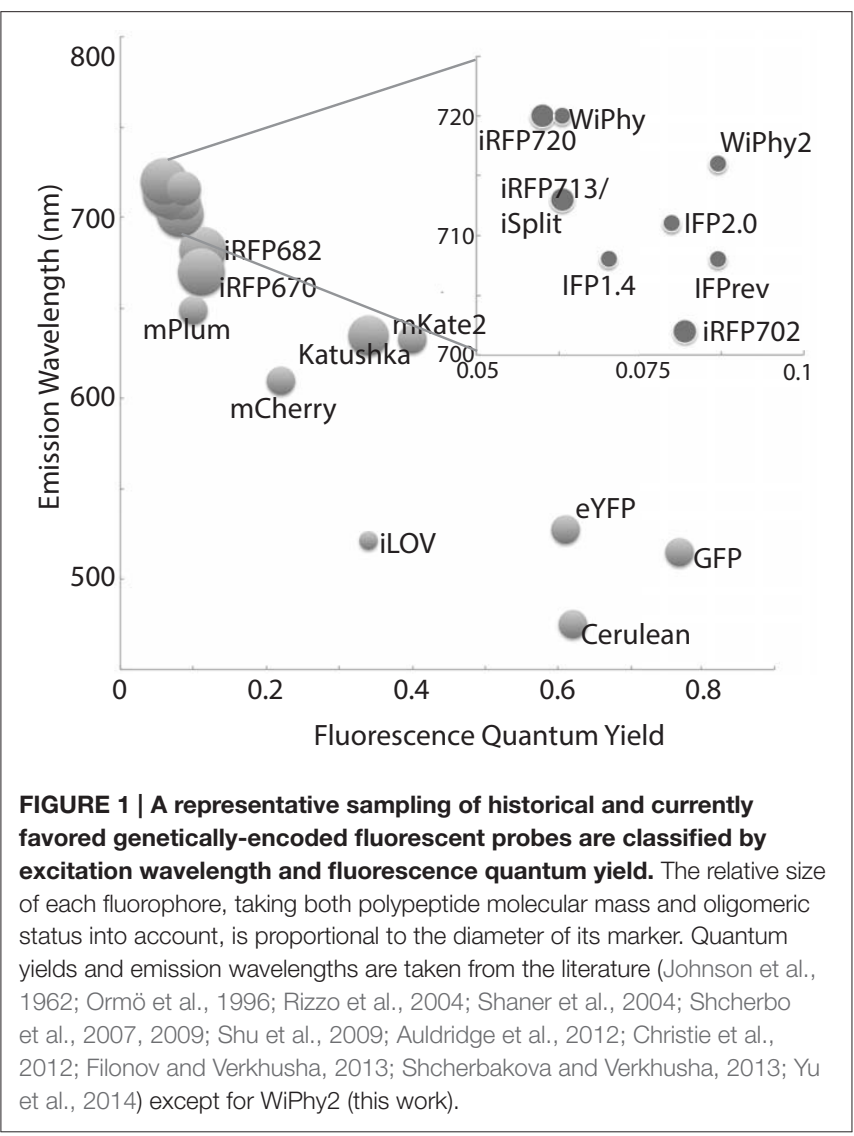


evidence for PPIX $\alpha$ binding includes the fact that fluorescence spectroscopy of the D207A apoprotein assembled with BV detected two fluorescent species, one matching the absorption and emission spectra of incorporated PPIX $\alpha$, and a second matching those for BV (Fischer and Lagarias, 2004; Lehtivuori et al., 2013).

In this paper we engineered two $\operatorname{DrCBD}_{\text {mon }}$ variants containing D207L; DrCBD ${ }_{\text {mon }}-\mathrm{D} 207 \mathrm{~L}$ itself and $\mathrm{DrCBD}_{\text {mon }}{ }^{-}$ Y263F/D207L (WiPhy2). We present a detailed comparative analysis of the spectroscopic properties of these two variants, as well as the three-dimensional structure of WiPhy2. This structural and spectroscopic study improves the integrated understanding of the fluorescence properties of BphPs.

\section{MATERIALS AND METHODS}

\section{Cloning}

Unless otherwise indicated, all reagents and solvents were obtained from commercial suppliers and used without further purification. Novel constructs were made by QuickChange mutagenesis (Stratagene, La Jolla, CA) using an existing pET21a plasmid encoding $\operatorname{DrCBD}_{\text {mon }}$ with N-terminal T7 and C-terminal hexahistidine tags (Auldridge et al., 2012). The following primers were used to introduce the appropriate mutations: D207L: 5'TTTCCCGCGTCGCTCATTCCGGCGC AGGCC ${ }^{\prime}$; 5'TGCGCCGGAATGAGCGACGCGGGAAAAC GG3' and Y263F: 5'CATGCACATGCAGTTCCTGCGGAAC A3'; 5'CATGTTCCGCAGGAACTGCATGTGCA3'. Correct sequences of clones were verified using DNA sequencing at the University of Wisconsin-Madison Biotechnology Center.

\section{Protein Purification}

Constructs encoding $\mathrm{DrCBD}_{\text {mon }}$ variants were transformed into BL21 (DE3) expression cells and grown at $37^{\circ} \mathrm{C}$ in LB-amp $\left(0.1 \mathrm{mg} / \mathrm{ml}\right.$ ampicillin). At $\mathrm{OD}_{600} 0.5$, cells were induced with isopropyl- $\beta$-D-1-thiogalactopyranoside at $28^{\circ} \mathrm{C}$. Cells were harvested after $4 \mathrm{~h}$ by centrifugation at $5000 \times \mathrm{g}$ for $30 \mathrm{~min}$, resuspended in lysis buffer $(25 \mathrm{mM}$ Tris buffer, $\mathrm{pH} 8.0,50 \mathrm{mM} \mathrm{NaCl}, 5 \mathrm{mM}$ imidazole), and lysed by sonication. After clarification by centrifugation at $40,000 \times \mathrm{g}$ for $30 \mathrm{~min}$, the supernatant was incubated with a final concentration of $0.16 \mathrm{mM} \mathrm{BV}$ (Frontier Scientific Inc., Logan, UT) in the dark overnight. Proteins were affinity-purified under green light using nickel-nitrilotriacetic acid resin (Qiagen, Valencia, CA). Further purification was performed using hydrophobic interaction on a phenyl-Sepharose column (GE Healthcare) to separate apoand holophytochrome. Ammonium sulfate was added to the protein at a final concentration of $0.35 \mathrm{M}$ prior to loading. All buffers were filtered and degassed before use. Purified samples were dialyzed overnight against a 200 -fold excess volume of ( $30 \mathrm{mM}$ Tris. $\mathrm{HCl}, \mathrm{pH} 8.0$ ). Finally samples were concentrated to $20 \mathrm{mg} / \mathrm{ml}$, flash-vitrified, and stored at $-80^{\circ} \mathrm{C}$. Unless otherwise indicated, the samples were kept in the dark before and during the experiments.

\section{Structure Determination by X-Ray Crystallography}

Purified WiPhy2 protein was crystallized by hanging drop vapor diffusion with drops containing a 1:1 mixture of protein and reservoir solution (20\% PEG400 and $0.1 \mathrm{M}$ phosphate citrate buffer at $\mathrm{pH}$ 4.0). The crystal used for data collection at LSCAT was soaked for $5 \mathrm{~min}$ in a cryoprotectant of $20 \%$ glycerol in mother liquor before vitrification.

Data were collected at the Advanced Photon Source, beamline LSCAT 21-ID-D at a wavelength of $0.9787 \AA$ on a Mar 300 CCD detector. The data were integrated and scaled using HKL2000 (Table 1).

The structure was solved in two stages. First, an initial data set collected on a Bruker Microstar rotating anode/R6000 Proteum CCD detector setup from a crystal from the same crystallization

TABLE 1 | WiPhy2 X-ray data collection and structure determination statistics.

\begin{tabular}{|c|c|c|}
\hline & $\begin{array}{l}\text { Rotating anode } \\
\text { (PDB ID 4ZRR) }\end{array}$ & $\begin{array}{l}\text { LS-CAT ID-D } \\
\text { (PDB ID 4Z1W) }\end{array}$ \\
\hline \multicolumn{3}{|l|}{ DATA COLLECTION } \\
\hline Wavelength, $\AA$ & 1.5418 & 0.9785 \\
\hline Resolution*, $\AA$ & $47.60-1.50(1.60-1.50)$ & $23.8-1.30(1.35-1.30)$ \\
\hline Space Group & $\mathrm{C} 2$ & $\mathrm{C} 2$ \\
\hline Unit Cell [a, b, c $\left.(\stackrel{\AA}{)}), \beta\left(^{\circ}\right)\right]$ & $94.8,55.1,69.8,91.9$ & $94.4,53.3,65.7,90.9$ \\
\hline No. Unique Reflections & 57,159 & 80,665 \\
\hline No. Unique Reflections Obs. & 54,864 & 77,741 \\
\hline Completeness, \% & $96.0(91.2)$ & $96.5(94.3)$ \\
\hline Redundancy & $2.9(2.0)$ & $7.7(7.7)$ \\
\hline$<|/ \sigma|>$ & $14.69(4.83)$ & $33.2(4.0)$ \\
\hline Wilson B value, $\AA^{2}$ & 10.8 & 10.5 \\
\hline $\mathrm{R}_{\text {sym }}^{\dagger}, \%$ & $5.0(17.9)$ & $5.2(35.3)$ \\
\hline \multicolumn{3}{|l|}{ REFINEMENT } \\
\hline Resolution, $\AA$ & $25.0-1.50(1.54-1.51)$ & $23.22-1.30(1.33-1.30)$ \\
\hline $\mathrm{R}_{\text {work }} / \mathrm{R}_{\text {free }}, \neq \%$ & 16.0/19.5 (18.8/27.5) & $14.4 / 16.0(15.1 / 17.5)$ \\
\hline Rms deviations Bonds, $\AA$ & 0.007 & 0.006 \\
\hline 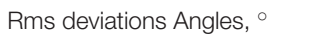 & 1.39 & 1.31 \\
\hline \multicolumn{3}{|l|}{ Ramachandran Statistics } \\
\hline Allowed & 98.6 & 98.0 \\
\hline Generously allowed & 1.4 & 2.0 \\
\hline \multicolumn{3}{|l|}{ No. Atoms } \\
\hline Protein & 2547 & 2551 \\
\hline Ligand & 86 & 86 \\
\hline Water & 288 & 231 \\
\hline \multicolumn{3}{|l|}{$<$ B factor $>, \AA^{2}$} \\
\hline Protein & 18.5 & 16.5 \\
\hline Ligand & 9.1 & 7.8 \\
\hline Water & 28.4 & 26.1 \\
\hline
\end{tabular}

${ }^{*}$ The highest resolution bin is indicated in parentheses.

$\dagger R_{\text {sym }}=\Sigma \Sigma j|l j-\langle 1\rangle| \Sigma \mid j$, where $l j$ is the intensity measurement for reflection $j$ and $\langle 1\rangle$ is the mean intensity for multiply recorded reflections.

${ }^{\ddagger} R_{\text {work }} / R_{\text {free }}=\Sigma|| F_{\text {obs }}|-| F_{\text {calc }}|||| F_{\text {obs }} \mid$, where the working and free $R$ factors are calculated by using the working and free reflection sets, respectively. For the $R_{\text {free }}, 5 \%$ of the total reflections were held aside throughout refinement. 
experiment was phased by molecular replacement using $4 \mathrm{O} 8 \mathrm{G}$ as a model, and refined. This structure was used to phase the higher resolution data set by molecular replacement using Phaser (McCoy et al., 2007). Those reflections assigned to the $\mathrm{R}_{\text {free }}$ bin were kept consistent.

For refinement, BV was linked to the Cys24 sulfur with a link entry in the input pdb. To create a library file for the ligand, BV was energy minimized using the Sybyl ${ }^{\circledR}-\mathrm{X}$ Suite
(Certara) employing the Tripos (Clark et al., 1989) Force Field after correct assignment of the atom types. The restraints for the two enantiomers of the chromophore (designated LBV and LBW) were generated using the Phenix (Adams et al., 2010) routine Elbow without energy minimization. Refinement and model building were carried out in iterative cycles using Refmac5.8.0107 and Coot V0.8.1 (Emsley et al., 2010; Murshudov et al., 2011). The BV chromophore is found with the A-ring C2

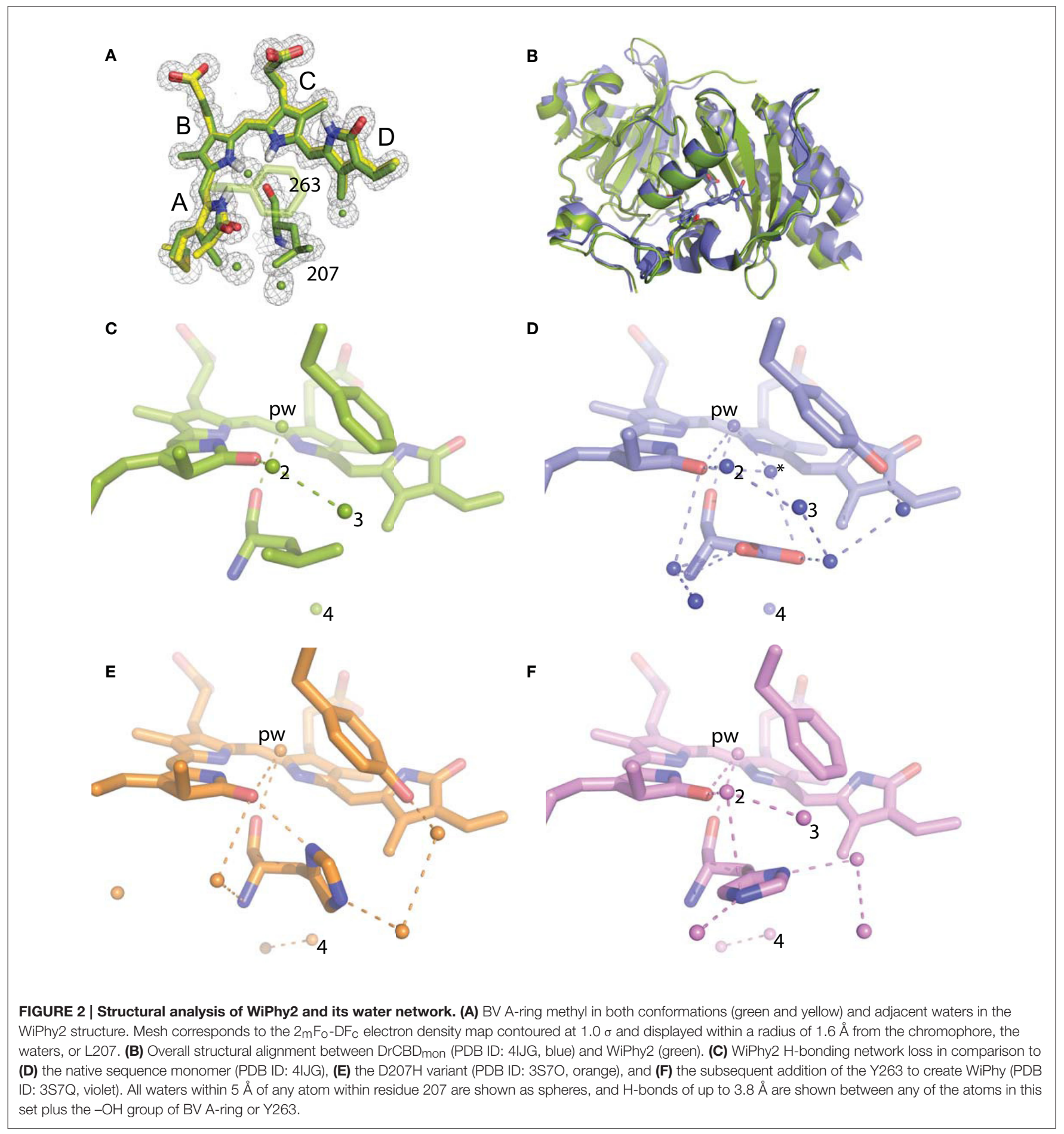


methyl occupying both up and down positions, consistent with previously published structures of DrCBD (Figure 2A).

\section{Spectroscopic Measurements}

All measurements were carried out at room temperature in complete darkness. Absorption wavelength scans in $1 \mathrm{~nm}$ steps from 250 to $850 \mathrm{~nm}$ were performed on a Beckman Coulter DU640B and Perkin Elmer LAMBDA 850 spectrophotometers. Sample illumination was as described previously (Auldridge et al., 2012). Briefly, samples were illuminated $15 \mathrm{~min}$ with red light or kept in the dark prior to each measurement. The $700 \mathrm{~nm}$ light was provided by a Fostec ACE light source fitted with a $700 \pm 5 \mathrm{~nm}$ interference filter (Andover Corp., Salem, NH). The light source-to-sample distance was adjusted so that irradiances of $150 \mu \mathrm{mol} \mathrm{m} \mathrm{m}^{-2} \mathrm{~s}^{-1}$ were used for $700 \mathrm{~nm}$ light.

The samples used to record steady-state and time-resolved fluorescence were diluted in $(30 \mathrm{mM}$ Tris. $\mathrm{HCl}, \mathrm{pH}$ 8.0) so that the absorption was sufficiently low $\left(\mathrm{OD}_{700}\right.$ close to 0.1 ) to prevent an inner filter effect. Fluorescence spectra were measured on a Tecan Infinite M1000 Monochromatorbased plate reader with a bandwidth of $5 \mathrm{~nm}$. Emission scans were run in Greiner FLUOTRAC 200 96-well flat-bottom black microplates. The excitation wavelength was $630 \mathrm{~nm}$. The excitation density was kept low to avoid photoconversion of the samples; its absence was confirmed by the identity of absorbance spectra immediately before and after the fluorescence experiments. The fluorescence quantum yields of $\mathrm{DrCBD}_{\text {mon- }}{ }^{-}$ D207L and WiPhy2 were determined relative to two reference fluorophores with known quantum yields (Eaton, 1988). Cy5-Nhydroxysuccinimidyl ester $\left(\Phi_{\mathrm{Cy} 5}=0.27\right.$; Lumiprobe) dissolved in phosphate-buffered saline (PBS) and Nile Blue perchlorate $\left(\Phi_{\text {NileBlue }}=0.27\right.$; Sigma Aldrich $)$ in acidic ethanol $[0.5 \%(\mathrm{v} / \mathrm{v})$ $0.1 \mathrm{M} \mathrm{HCl}$ in ethanol] were used as fluorescence quantum yield standards (Sens and Drexhage, 1981; Mujumdar et al., 1993). For $\mathrm{pH}$ titration experiments, the protein solution was diluted 50 -fold into the appropriate buffer $(\mathrm{pH} 4-7,30 \mathrm{mM}$ citrate-phosphate buffer; $\mathrm{pH} 7-9,30 \mathrm{mM}$ Tris- $\mathrm{HCl}$; and $\mathrm{pH} 9$ and 10, $30 \mathrm{mM}$ glycine). $\mathrm{pH}$ values of aqueous solutions were measured using a standard laboratory $\mathrm{pH}$ meter (Fisher Scientific ${ }^{\mathrm{TM}}$ ) calibrated prior to experiments using biotechnology grade standard buffer

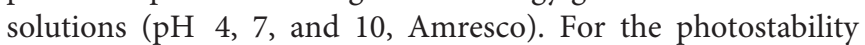
test for WiPhy2 the sample was continuously irradiated (696 \pm $5 \mathrm{~nm}$ ) within the Varian Cary Eclipse spectrophotometer. Power was $2.3 \mathrm{~mW}$, which corresponds to a photon flux of $160 \mu \mathrm{mol}$ $\mathrm{m}^{-2} \mathrm{~s}^{-1}$. Fluorescence intensity was measured at $719 \mathrm{~nm}$ every $5 \mathrm{~min}$.

The excitation-emission matrix (EEM) of $\mathrm{DrCBD}_{\text {mon }}-\mathrm{D} 207 \mathrm{~L}$ and WiPhy2 were recorded on a Varian Cary Eclipse fluorescence spectrophotometer. The EEM fluorescence spectrum was obtained by concatenating emission spectra measured from 630 to $850 \mathrm{~nm}$ by using excitation wavelengths of $550-770 \mathrm{~nm}(5 \mathrm{~nm}$ intervals) with $0.1 \mathrm{~s}$ integration time and a $5 \mathrm{~nm}$ slit widths. The Raman scattering peaks in the EEM spectrum were corrected with a described method (Zepp et al., 2004). The sample resided in a vertically mounted glass capillary with an inner diameter close to $1.1 \mathrm{~mm}$ (VITREX, micro-haematocrit) with $\mathrm{OD}_{700}$ of about $0.1 / \mathrm{mm}$. To avoid excessive sample degradation, the sample solution (volume $400 \mu \mathrm{L}$ ) was cycled using a peristaltic pump (Ismatec, Reglo Digital) at a flow rate of $0.1 \mathrm{~mL} \mathrm{~min}^{-1}$ through a glass reservoir, the capillary, and connecting Teflon tubing ( $1 \mathrm{~mm}$ inner diameter). A far-red laser diode (750 \pm $5 \mathrm{~nm}, 3 \mathrm{~mW}$, Leading-Tech Laser Co.) was used to transform the sample to the Pr state by constantly illuminating the sample through the Teflon tubing.

Fluorescence decays of the samples in the sub-nanosecond and nanosecond time scales were measured using a time-correlated single photon counting (TCSPC) system consisting of a HydraHarp 400 controller and a PDL 800-B driver (PicoQuant $\mathrm{GmBH}$ ). The excitation wavelength was $660 \mathrm{~nm}$ from a pulsed diode laser head LDH-P-C-660. The repetition rate of the excitation pulses was set to $40 \mathrm{MHz}$ in all measurements, and the output power of the laser was $0.98 \mathrm{~mW}$ for $660 \mathrm{~nm}$ excitation. The Jobin Yvon monochromator was used to detect the emission at $720 \mathrm{~nm}$ with a single photon avalanche photodiode (SPAD, MPD-1CTC). The time resolution was approximately 70 ps [full width at half-maximum of the instrument response function (IRF)]. The data were fitted with monoexponential functions to obtain fluorescence lifetimes (Lehtivuori et al., 2013). In addition to the fluorescence decay components, a fast rise component of about 20 ps was needed to obtain satisfactory fits at early time points.

\section{RESULTS}

\section{Structural Properties}

To gain insight into the fluorescent nature of WiPhy2, a 1.3 $\AA$ resolution crystal structure was obtained (Table 1). There were no significant changes to the overall structure of WiPhy2 compared to $\mathrm{DrCBD}_{\text {mon }}$ (RMSD $0.82 \AA$ over all 296 shared $\mathrm{C} \alpha$ atoms including mobile loop regions; Figure 2B). The BV chromophore is well-ordered with no evidence of a break in electron density for the cysteine connection to the A-ring (Figure 2A).

The most obvious result from this new structure is the confirmation of our hypothesis: waters are less abundant around the L207 side chain than has been seen in other high-resolution structures containing either Asp or His at this position. For D207L, within $5 \AA$ of any Leu atom there are only four waters, including the pyrrole water with strong interactions to $\mathrm{BV}$ nitrogen atoms in A-, B-, and C-rings (Figure 2C). The closest of these waters to any Leu side chain atom is $3.8 \AA$. All four water positions are conserved in the water network of DrCBD $_{\text {mon }}$ (PDB ID: 4IJG, Figure 2D; Bhattacharya et al., 2014). The second and third form a path from the pyrrole water to the solvent, whereas the fourth is located under the residue 207 side chain and forms a H-bond with Y176. Of course, there are no H-bonds from any of these waters to the L207 side chain in WiPhy2.

This paucity of solvent molecules can be strongly contrasted with the native sequence found in the monomer structure, which holds nine waters (Figure 2D). These waters permit an extensive H-bonding network of 16 different pair wise interactions of $3.8 \AA$ or less between any two atoms in the set containing all atoms in 
residue 207, the $-\mathrm{OH}$ of $\mathrm{Y} 263$, the $-\mathrm{OH}$ of the A-ring, and these waters.

The introduction of His at position 207 (PDB ID: 3S7O; Auldridge et al., 2012) diminishes this H-bonding network somewhat, with seven waters and nine remaining $\mathrm{H}$-bonds (Figure 2E). For WiPhy (PDB ID: 3S7Q; (Auldridge et al., 2012)), which also has the polar His at position 207 but introduces the nonpolar Phe263, there remain eight waters in the $5 \AA$ cutoff window from the 207 side chain and $9 \mathrm{H}$-bonds (Figure 2F). Thus, we can conclude that the identity of the residue at position 207 and not at position 263 has the greatest effect on this water network near the "mouth" leading from BV to the solvent. Among the four structures compared here, it is notable that only in $\mathrm{DrCBD}_{\text {mon }}$ which is known to weakly photoconvert (Auldridge et al., 2012) is there a second water located between the chromophore and this outlet (Figure 2D, asterisk).

\section{Spectroscopic Properties}

In the dark state (Figure 3), both of the D207L constructs show absorption spectra in the characteristic phytochrome region between 600 and $800 \mathrm{~nm}$ similar to other monomeric $\mathrm{DrCBD}_{\text {mon }}$ variants (Auldridge et al., 2012; Bhattacharya et al., 2014; Takala et al., 2015). The D207L constructs have absorption maxima at 696 (WiPhy2) and $697\left(\mathrm{DrCBD}_{\mathrm{mon}} \mathrm{D} 207 \mathrm{~L}\right)$, respectively, with a pronounced shoulder at $650 \mathrm{~nm}$. The slight shift $(-2 \mathrm{~nm}$ relative to $\mathrm{DrCBD}_{\text {mon }}$ ) in the maximum absorption wavelength is a hypsochromic shift caused by the decreased hydrogen-bond network around $\mathrm{BV}$ compared to $\mathrm{DrCBD}_{\text {mon }}$ and WiPhy. The amplitude of the shoulder at $650 \mathrm{~nm}$ (Figure 3, inset) is higher in the case of the proteins containing Leu at position 207 compared to previously analyzed derivatives of $\mathrm{DrCBD}_{\text {mon }}$.

The photoconversion potentials for both D207L variants were also tested (Figure 4). After illumination with red light, the Q

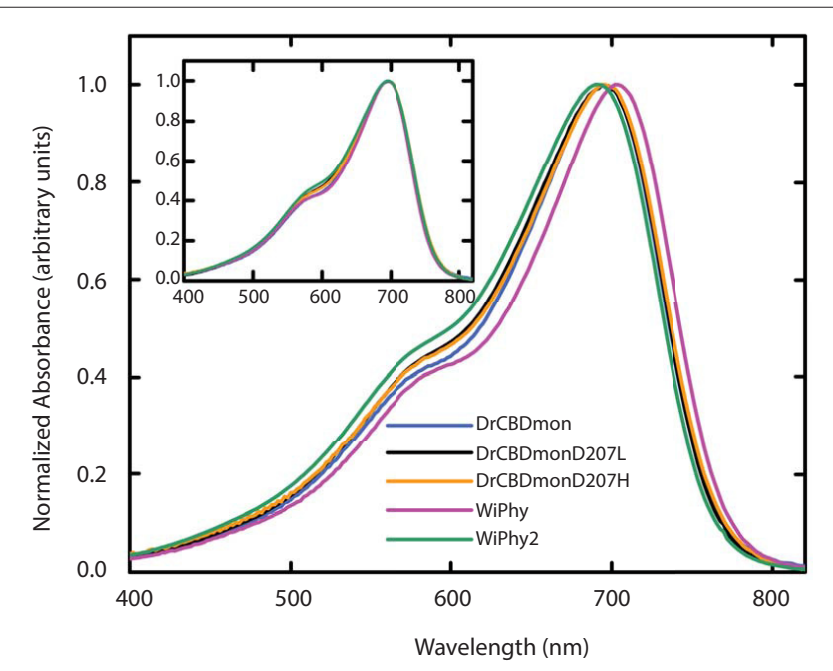

FIGURE 3 | UV-Vis absorption spectra of the five $\operatorname{DrCBD}_{\text {mon }}$ variants in the Pr state immediately after sample thawing. All absorption spectra were normalized at their maxima. (Inset) Spectra are aligned at their absorbance maxima for better visualization of shoulder heights. band at $696 \mathrm{~nm}$ decreases (Figure 4, inset) in each. While native $\mathrm{BphP}$ and $\mathrm{DrCBD}_{\text {mon }}$ as well as other studied variants show an increase in absorbance at $750 \mathrm{~nm}$ upon illumination (Auldridge et al., 2012), neither D207L sample has this behavior. Instead, in the two D207L-containing samples, there is a noticeable increase in absorbance in the photoproduct at $730 \mathrm{~nm}$ (Figure 4, inset). This difference implies an incomplete or a different type of photocycle than other $\operatorname{DrCBD}_{\text {mon }}$ variants.

The fluorescence spectra of the D207L samples, when excited at the Q-band of BV at $630 \mathrm{~nm}$, are presented in Figure 5A. The observed fluorescence emission originates from the $\mathrm{BV}$ chromophore, with the same spectral shape in $\operatorname{DrCBD}_{\text {mon }} \mathrm{D} 207 \mathrm{~L}$ and WiPhy2, in keeping with their matching absorption spectra (Figure 3). The maxima of the emission spectra are located at 722 and $719 \mathrm{~nm}$ for $\mathrm{DrCBD}_{\text {mon }} \mathrm{D} 207 \mathrm{~L}$ and WiPhy2, respectively. Their fluorescence quantum yields were determined to be $0.070 \pm 0.005\left(\mathrm{DrCBD}_{\mathrm{mon}} \mathrm{D} 207 \mathrm{~L}\right)$ and $0.087 \pm 0.005$ (WiPhy2). The quantum yield of WiPhy2 is thus $24 \%$ higher than that of $\operatorname{DrCBD}_{\text {mon }}$ D207L.

A similar trend was observed for the time-resolved lifetime measurements (Figure 5B). Using excitation wavelength of $660 \mathrm{~nm}$ and monitoring wavelength of $720 \mathrm{~nm}$, the excitation decay properties of $\mathrm{BV}$ molecules in the binding pocket can be studied. The excited state decay can be described by monoexponential components, with time constants of $650 \pm 30$ ps for $\mathrm{DrCBD}_{\text {mon }} \mathrm{D} 207 \mathrm{~L}$ and $780 \pm 30$ ps for WiPhy2 (parameters summarized in Table 2).

The emission-excitation matrix (EEM) spectra for both D207L variants were measured to study with finer detail how fluorescence properties vary with excitation wavelength. We sought to determine whether WiPhy2 binds PPIX $\alpha$ present naturally in the cells it is expressed in. Given that the shape of the fluorescence spectra remains the same in every

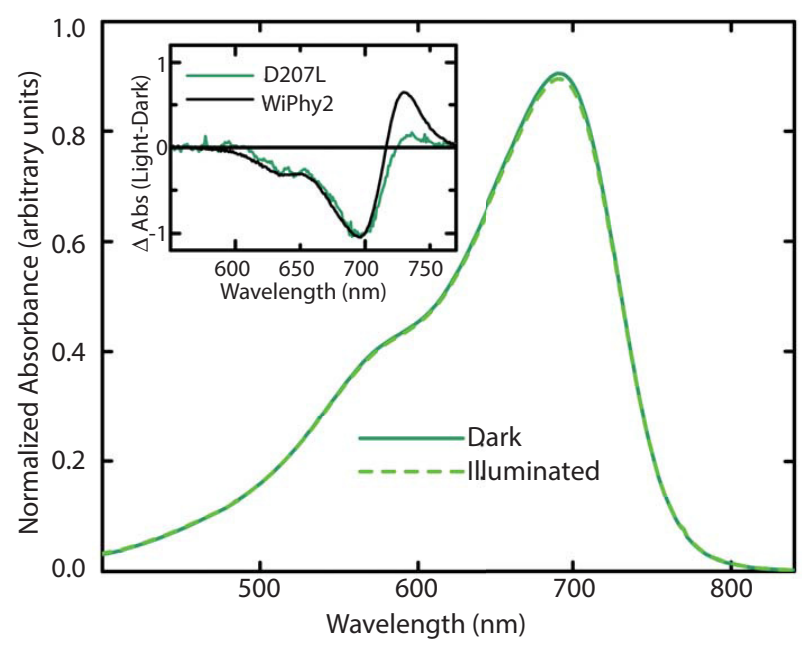

FIGURE 4 | Steady-state absorption spectra of purified WiPhy2 measured immediately upon thawing (dark) and after $15 \mathrm{~min}$ of $700 \mathrm{~nm}$ light illumination (illuminated). Inset shows absorption difference spectra of DrCBD $_{m o n}$ D207L and WiPhy2 (red irradiated spectrum has been subtracted from dark spectrum) absorption difference spectra were normalized at $698 \mathrm{~nm}$. 


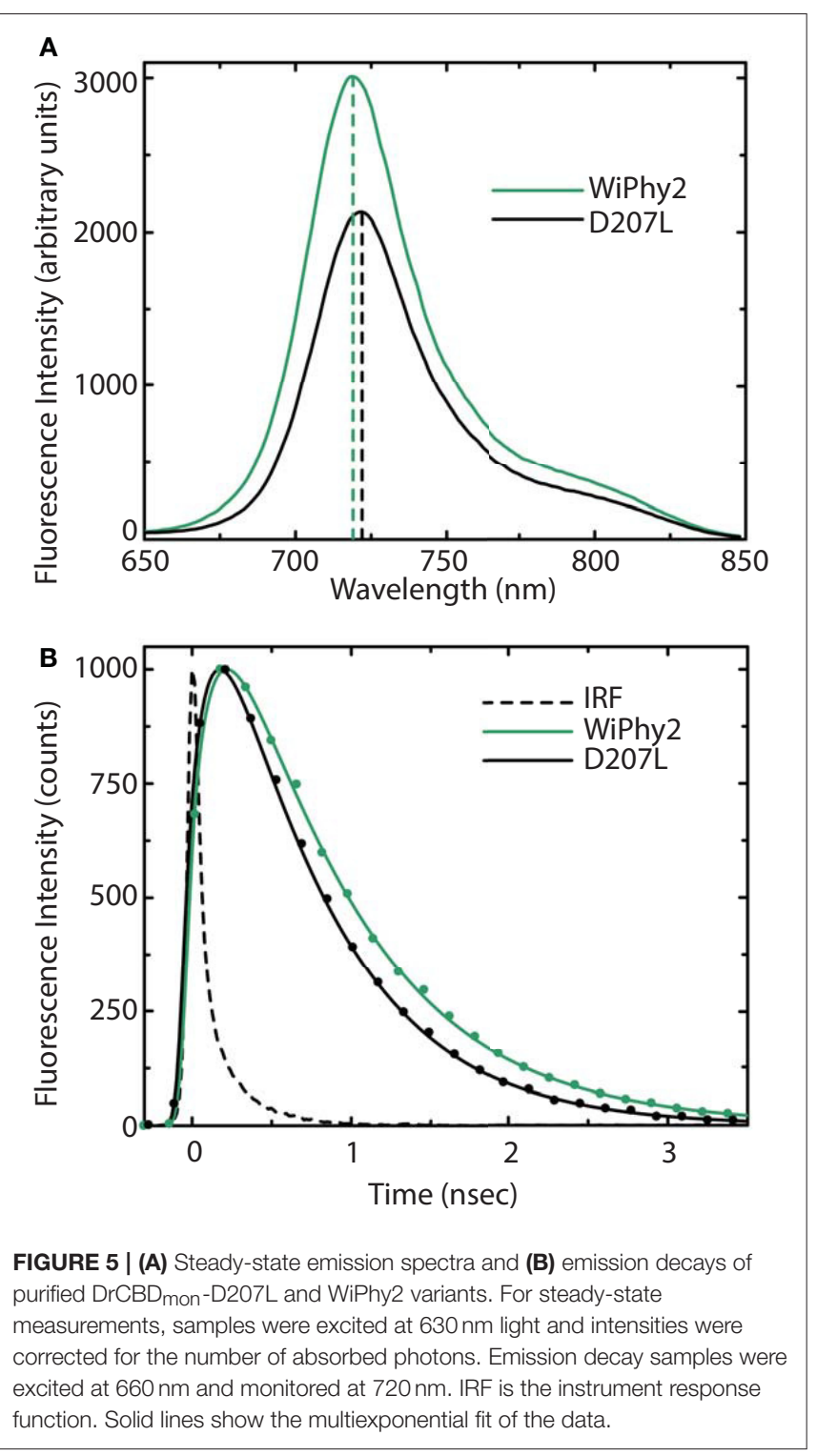

TABLE 2 | Quantum yield measurements and fluorescence lifetimes.

\begin{tabular}{|c|c|c|c|c|c|}
\hline $\begin{array}{l}\text { Protein } \\
\text { variant }\end{array}$ & $\begin{array}{c}\text { Abs. } \\
\max (n m)\end{array}$ & $\begin{array}{c}\text { Em. } \\
\max (n m)\end{array}$ & $\Phi(\%)$ & $\begin{array}{l}\text { Lifetime } \\
\text { (ps) }\end{array}$ & $\begin{array}{l}\text { Binding } \\
\text { PPIX } \alpha\end{array}$ \\
\hline $\begin{array}{l}\text { DrCBD mon- } \\
\text { D207L }\end{array}$ & 697 & 722 & $7.0 \pm 0.5$ & $\begin{array}{c}650 \pm 30 \\
\text { ps }\end{array}$ & ND \\
\hline $\begin{array}{l}\text { WiPhy2 } \\
\text { (DrCBDmon- } \\
\text { Y263F/D207L }\end{array}$ & 696 & 719 & $8.7 \pm 0.5$ & $\begin{array}{c}780 \pm 30 \\
\text { ps }\end{array}$ & No \\
\hline
\end{tabular}

excitation wavelength (Figures 6A,B), in contrast, for example, to $\mathrm{DrCBD}_{\text {mon }}$, we conclude that the fluorescence emission from WiPhy2 originates only from the BV chromophore. The composite EEM reveals the change in fluorescence intensity as a function of wavelength and shows a single maximum at ex $=$ 696, em $=719 \mathrm{~nm}$ (Figure 6A).
We further investigated the effect of $\mathrm{pH}$ on absorption and fluorescence of WiPhy2 (Figure 7A). The fluorescence intensity of sample displayed linear responses to $\mathrm{pH}$ values in the range from 4 to 9. The sample was non-fluorescent with $\mathrm{pH}>$ 9. Correspondingly, the absorption spectra of WiPhy2 was unaffected by changes in $\mathrm{pH}$ ranging from 4.0 to 9.0 (Figure 7A, inset). Drop-off in fluorescence at low $\mathrm{pH}$ has been noted with some of the previously published fluorescent variants with His in the 207th position (Filonov et al., 2011) likely due to changes in the protonation state of His ( $\mathrm{pKa}$ 6.1). This challenge is removed in the case of L207.

Photostability of the WiPhy2 variant was also tested. Samples were excited continuously at their optimal excitation wavelength $(696 \mathrm{~nm})$ and fluorescence intensity was measured at $719 \mathrm{~nm}$ every $5 \mathrm{~min}$. Fluorescence dropped by only $2 \%$ after $60 \mathrm{~min}$ (Figure 7B).

\section{DISCUSSION}

Bacteriophytochromes are characterized by structural and spectroscopic variability. Intermediates in their light-driven forward and backward reactions have been trapped at low temperature and spectrally characterized (Eilfeld and Rüdiger, 1985). The first, formed from the excited state bilin molecule on a timescale of ps-ns, is the Lumi-R state. In BphPs, Lumi-R has a ground-state bleach at $700 \mathrm{~nm}$ and induced absorption at $730 \mathrm{~nm}$ (Toh et al., 2011b). None of the fulllength DrBphP Asp207 substitutions stably photoconvert to Pfr upon photoexcitation with red light, although some do reach the Meta-R state, with a steady-state absorption peak between 740 and $750 \mathrm{~nm}$ (Borucki et al., 2005; Wagner et al., 2008). By reduction of polarity near the chromophore of WiPhy, we endeavored to limit photoconversion and excited state proton transfer and thus improve fluorescence yield. This goal was informed by the fact that for full-length dimeric DrBph-D207L there is essentially no steady state photoconversion even after extended irradiation (Wagner et al., 2008), and by the fact that two phytofluors in the iRFP series carry D207L substitutions among others.

Here we show that $\mathrm{DrCBD}_{\text {mon }} \mathrm{D} 207 \mathrm{~L}$ and WiPhy2 respond to red light with miniscule decreases in absorption at $696 \mathrm{~nm}$ and induced absorption at $730 \mathrm{~nm}$ (Figure 4, inset). Logically, there is also no subsequent appearance of absorbance at $750 \mathrm{~nm}$ to indicate the Meta-R or Pfr photoproducts as seen in the parent $\mathrm{DrCBD}_{\text {mon }}$. Formation of the Meta-R state must require the polar hydrogen-bonding network that is set up by the chromophore, charged residue at position 207, and associated waters (Figure 2). Thus, our study reinforces the major role of Asp207 in the photocycle, and demonstrates that Leu at this position leads to negligible Lumi-R photoproduct yield. Since only steadystate measurements were carried out, we are unable to draw conclusions about a particular reaction scheme for the excited state Pr to the Lumi-R-state. We demonstrate that WiPhy2 has a fluorescence quantum yield of nearly $9 \%$ and an excited state lifetime of 780 ps, both $\sim 20 \%$ higher than WiPhy (Figure 5), representing the effect of the Leu on the excited state decay of $\mathrm{BV}$ in the binding pocket. 

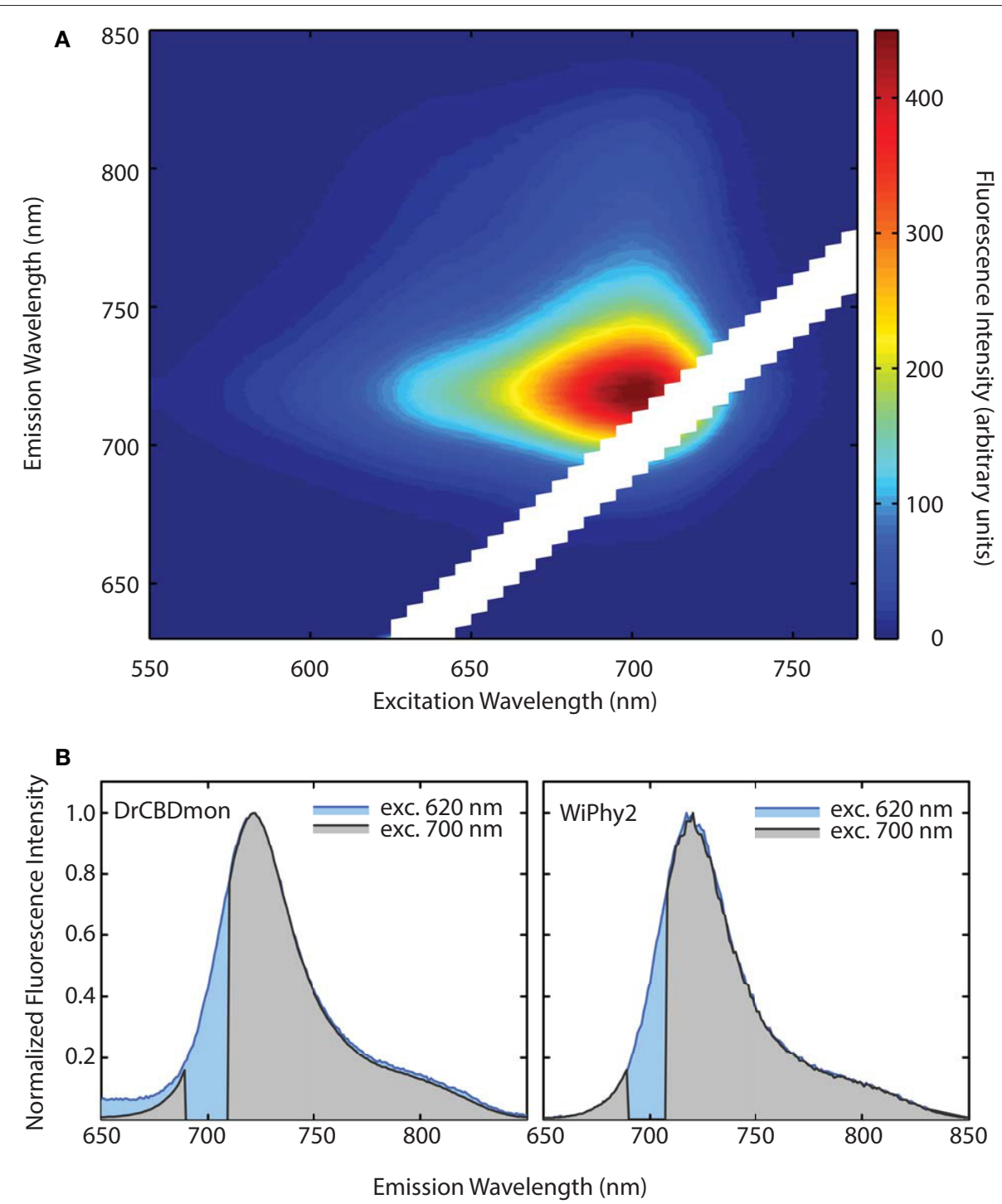

FIGURE 6 | (A) Excitation-Emission Matrix (EEM) for WiPhy2 represents fluorescence as a function of both excitation and emission wavelengths. Fluorescence intensities are corrected with the number of absorbed photons. (B) Two extracted excitation wavelengths $(620 \mathrm{~nm}$ and $700 \mathrm{~nm})$ are compared for DrCBDmon (left) and WiPhy2 (right).

The excited state lifetimes for both fluorescent L207 $\mathrm{DrCBD}_{\text {mon }}$ variants $(650$ and 780 ps) are longer than for DrCBDmon (620 ps; Bhattacharya et al., 2014) or Rhodopseudomonas palustris BphP3 (362 ps; Toh et al., 2011b). Although WiPhy2 lifetime was increased compared to $\mathrm{DrCBD}_{\text {mon }}$, cyanobacterial Cph1 variant lifetimes have been reported as long as $1.8 \mathrm{~ns}$ and $\mathrm{IFP}_{\text {rev }}$ has a lifetime of 815 ps at room temperature (Miller et al., 2006; Bhattacharya et al., 2014; Kim et al., 2014a). One key reason for variation in lifetimes, apart from the slightly different bilin in Cph1, is immobilization of the D-ring by H-bonding and/or hydrophobic packing (Toh et al., 2011b; Bhattacharya et al., 2014).

We now show that a second mechanism for achieving a longer decay lifetime is to decrease the number of waters near the chromophore, which in turn curtails the hydrogen-bonding network among BV, polar side chains and coordinating water molecules. Opportunities for photoconversion or excited state proton transfer are thus lost. DrCBD variants analyzed to date have clear interactions among solvent molecules (Auldridge et al., 2012; Bhattacharya et al., 2014) but in WiPhy2 there are fewer waters than in any other structurally characterized $\operatorname{DrCBD}_{\text {mon }}$ variant (Figure 2). Thus, the change to Leu might be viewed as a "mutation" of waters away from the chromophore. We note that the crystals from which these data were obtained have the same C2 space group and are grown under similar solvent content and $\mathrm{pH}$, lending validity to the comparisons. Nonetheless, because cryopreservation conditions may affect overall solvent distributions and because moreover for our spectroscopic studies solution water molecules can be expected to exchange rapidly, our conclusion focuses on this trend rather 

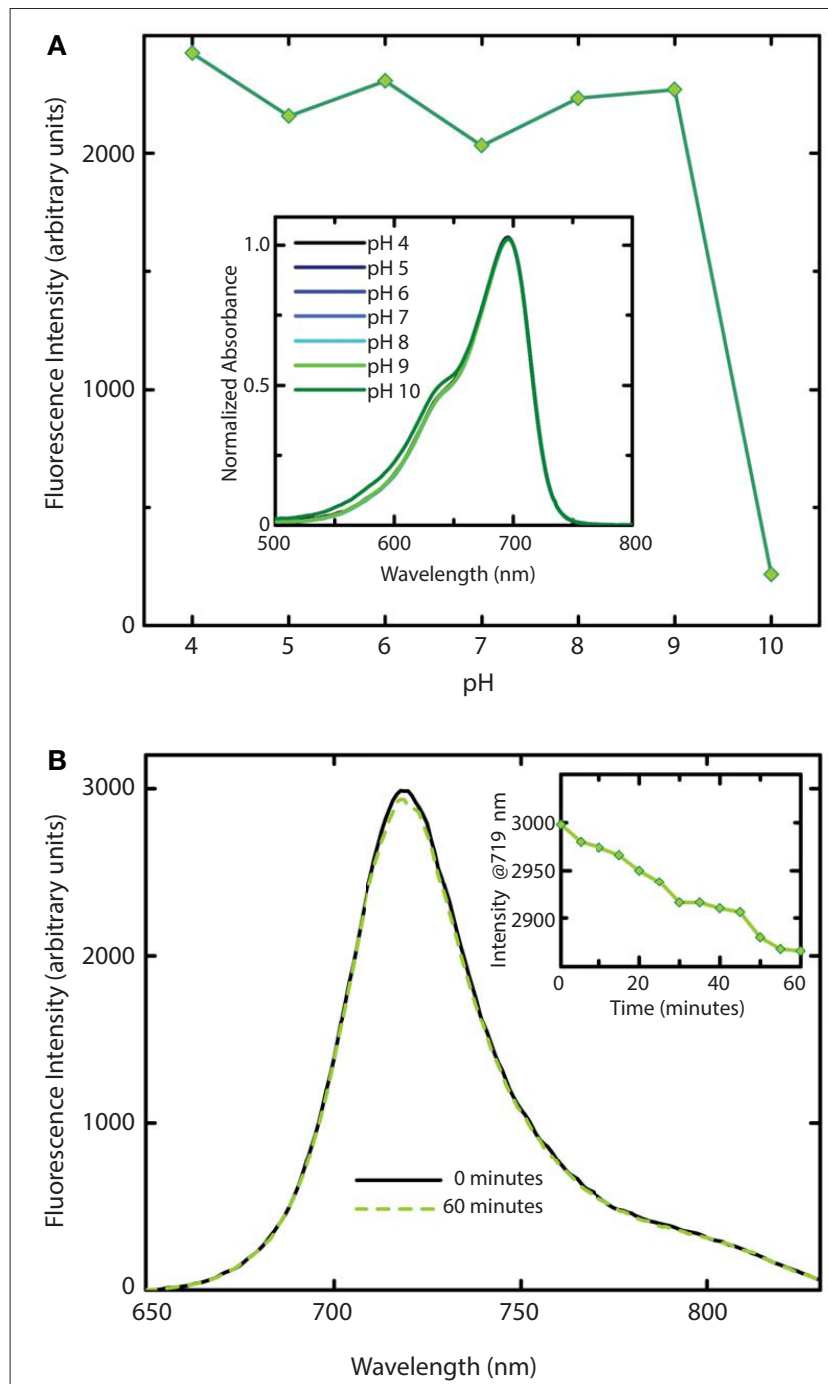

FIGURE 7 | Stability of WiPhy2 Fluorescence vs. pH and illumination time. (A) Dependency of WiPhy2 absorption (inset) and fluorescence on $\mathrm{pH}$. (B) Steady-state emission spectra of WiPhy2 before and after a 1-h photostability test. Inset shows fluorescence intensity at peak over time.

than a particular water constellation. Following the same logic, our conclusions are not affected by the existence of heterogeneity in the ground or excited states (Samma et al., 2010; Song et al., 2011; Kim et al., 2014b). Thus, X-ray crystallography confirmed the spatial observations of spectroscopic studies; removing polar interactions in the vicinity of the chromophore shifts steady state absorption band location, photocycle yields and fluorescence properties.

\section{REFERENCES}

Adams, P. D., Afonine, P. V., Bunkóczi, G., Chen, V. B., Davis, I. W., Echols, N., et al. (2010). PHENIX: a comprehensive Python-based system for macromolecular structure solution. Acta Crystallogr. D Biol. Crystallogr. 66, 213-221. doi: 10.1107/S0907444909052925
Previously it has been demonstrated that phytochromes' absorbance shoulder around $650 \mathrm{~nm}$ is not due to a second chromophore species (such as PPIX $\alpha$ ) but is instead a natural physical consequence of vibronic progressions in the absorption spectrum (Spillane et al., 2009, 2012). PPIX $\alpha$ binding to the CBD has been a noted disadvantage in several described phytofluors. In the case of $\operatorname{DrBphP} \mathrm{D} 207 \mathrm{H}$ and as first described in cyanobacterial phytochrome variants (Cph1 Y176R in particular) by Lagarias and coworkers, not only the linear chromophore but also cyclized PPIX $\alpha$ is accommodated in the binding pocket (Fischer and Lagarias, 2004), as observed in emission spectra upon excitation at $600-650 \mathrm{~nm}$. Lehtivuori et al. have shown these minor emission bands due to PPIX $\alpha$ at $660 \mathrm{~nm}$ in DrCBD$\mathrm{D} 207 \mathrm{H}$ as well as to some extent in DrCBD (Lehtivuori et al., 2013). Burgie et al. obtained the same result in the case of DrCBD and its D207A variant (Burgie et al., 2014). For both of our D207L variants this PPIX $\alpha$ binding disadvantage is alleviated, as seen in our EEM spectra. Indeed, regardless of the excitation maximum, we obtain same emission spectra of only BV (Figure 7).

We have used steady state and time-resolved spectroscopy as well as protein crystallography to test the hypothesis that the introduction of a nonpolar amino acid in place of the native charge in DrCBD would improve its fluorescence properties. Indeed, in WiPhy2 Leu at position 207 raises fluorescence quantum yield and lengthens excited state lifetime, maintaining an illumination wavelength of nearly $700 \mathrm{~nm}$ while avoiding PPIX $\alpha$ binding or a narrow $\mathrm{pH}$ window for efficacy (Figure 1).

\section{FUNDING}

The research was supported by the Academy of Finland grant 277194 (HL), University of Jyväskylä (HL), the Fulbright Center in Finland (HL), the National Science Foundation 1518160 (KTF), and the W. H. Peterson Fellowship (SB). Use of the Advanced Photon Source, an Office of Science User Facility operated for the U.S. Department of Energy (DOE) Office of Science by Argonne National Laboratory, was supported by the U.S. DOE under Contract No. DE-AC02-06CH11357. Use of the LS-CAT Sector 21 was supported by the Michigan Economic Development Corporation and the Michigan Technology TriCorridor (Grant 085P1000817).

\section{ACKNOWLEDGMENTS}

The high resolution WiPhy2 structure discussed here has been deposited in the Protein Data Bank with accession code PDB ID: $4 Z 1 W$. In addition the lower resolution structure solved with rotating anode data has been deposited with code PDB ID: 4ZRR. 
Interdiscip. Rev. Nanomed. Nanobiotechnol. doi: 10.1002/wnan.1351. [Epub ahead of print].

Bhattacharya, S., Auldridge, M. E., Lehtivuori, H., Ihalainen, J. A., and Forest, K. T. (2014). Origins of fluorescence in evolved bacteriophytochromes. J. Biol. Chem. 289, 32144-32152. doi: 10.1074/jbc.M114.589739

Borucki, B., von Stetten, D., Seibeck, S., Lamparter, T., Michael, N., Mroginski, M. A., et al. (2005). Light-induced proton release of phytochrome is coupled to the transient deprotonation of the tetrapyrrole chromophore. J. Biol. Chem. 280, 34358-34364. doi: 10.1074/jbc.M5054 93200

Burgie, E. S., Wang, T., Bussell, A. N., Walker, J. M., Li, H., and Vierstra, R. D. (2014). Crystallographic and electron microscopic analyses of a bacterial phytochrome reveal local and global rearrangements during photoconversion. J. Biol. Chem. 289, 24573-24587. doi: 10.1074/jbc.M114. 571661

Christie, J. M., Hitomi, K., Andrew, A. S., Hartfield, K. A., Mettlen, M., Pratt, A. J., et al. (2012). Structural tuning of the fluorescent protein ilov for improved photostability. J. Biol. Chem. 287, 22295-22304. doi: 10.1074/jbc.M111.318881

Clark, M., Cramer, R. D. III, and Van Opdenbosch, N. (1989). Validation of the general purpose tripos 5.2 force field. J. Comput. Chem. 10, 982-1012. doi: $10.1002 /$ jcc. 540100804

Eaton, D. F. (1988). Reference materials for fluorescence measurements. Pure Appl. Chem. 60, 1107-1114.

Eilfeld, P., and Rüdiger, W. (1985). Absorption spectra of phytochrome intermediates. Z. Naturforsch. C 40, 109-113.

Emsley, P., Lohkamp, B., Scott, W. G., and Cowtan, K. (2010). Features and development of Coot. Acta Crystallogr. D Biol. Crystallogr. 66, 486-501. doi: $10.1107 /$ S0907444910007493

Escobedo, J. O., Rusin, O., Lim, S., and Strongin, R. M. (2010). NIR dyes for bioimaging applications. Curr. Opin. Chem. Biol. 14, 64-70. doi: 10.1016/j.cbpa.2009.10.022

Filonov, G. S., and Verkhusha, V. V. (2013). A near-infrared bifc reporter for in vivo imaging of protein-protein interactions. Chem. Biol. 20, 1078-1086. doi: 10.1016/j.chembiol.2013.06.009

Filonov, G. S., Piatkevich, K. D., Ting, L., Zhang, J., Kim, K., and Verkhusha, V. V. (2011). Bright and stable near-infrared fluorescent protein for in vivo imaging. Nat. Biotechnol. 29, 757-761. doi: 10.1038/nbt.1918

Fischer, A. J., and Lagarias, J. C. (2004). Harnessing phytochrome's glowing potential. Proc. Natl. Acad. Sci. U.S.A. 101, 17334-17339. doi: 10.1073/pnas.0407645101

Gibbs, S. L. (2012). Near infrared fluorescence for image-guided surgery. Quant. Imaging Med. Surg. 2, 177-187. doi: 10.3978/j.issn.2223-4292.2012.09.04

Guo, Z., Park, S., Yoon, J., and Shin, I. (2014). Recent progress in the development of near-infrared fluorescent probes for bioimaging applications. Chem. Soc. Rev. 43, 16-29. doi: 10.1039/C3CS60271K

Hahn, M. A., Singh, A. K., Sharma, P., Brown, S. C., and Moudgil, B. M. (2011). Nanoparticles as contrast agents for in-vivo bioimaging: current status and future perspectives. Anal. Bioanal. Chem. 399, 3-27. doi: 10.1007/s00216-0104207-5

Johnson, F. H., Shimomura, O., Saiga, Y., Gershman, L. C., Reynolds, G. T., and Waters, J. R. (1962). Quantum efficiency of Cypridina luminescence, with a note on that ofAequorea. J. Cell. Comp. Physiol. 60, 85-103. doi: 10.1002/jcp.1030600111

Kim, P. W., Rockwell, N. C., Martin, S. S., Lagarias, J. C., and Larsen, D. S. (2014a). Dynamic inhomogeneity in the photodynamics of cyanobacterial phytochrome Cph1. Biochemistry 53, 2818-2826. doi: 10.1021/bi500108s

Kim, P. W., Rockwell, N. C., Martin, S. S., Lagarias, J. C., and Larsen, D. S. (2014b). Heterogeneous photodynamics of the pfr state in the cyanobacterial phytochrome Cph1. Biochemistry 53, 4601-4611. doi: 10.1021/bi5005359

Lehtivuori, H., Rissanen, I., Takala, H., Bamford, J., Tkachenko, N. V., and Ihalainen, J. A. (2013). Fluorescence properties of the chromophore-binding domain of bacteriophytochrome from Deinococcus radiodurans. J. Phys. Chem. B 117, 11049-11057. doi: 10.1021/jp312061b

Luo, S., Zhang, E., Su, Y., Cheng, T., and Shi, C. (2011). A review of NIR dyes in cancer targeting and imaging. Biomaterials 32, 7127-7138. doi: 10.1016/j.biomaterials.2011.06.024

Marx, V. (2014). Probes: seeing in the near infrared. Nat. Methods 11, 717-720. doi: $10.1038 /$ nmeth.3001
McCoy, A. J., Grosse-Kunstleve, R. W., Adams, P. D., Winn, M. D., Storoni, L. C., and Read, R. J. (2007). Phaser crystallographic software. J. Appl. Crystallogr. 40, 658-674. doi: 10.1107/S0021889807021206

Miller, A. E., Fischer, A. J., Laurence, T., Hollars, C. W., Saykally, R. J., Lagarias, J. C., et al. (2006). Single-molecule dynamics of phytochrome-bound fluorophores probed by fluorescence correlation spectroscopy. Proc. Natl. Acad. Sci. U.S.A. 103, 11136-11141. doi: 10.1073/pnas.0604724103

Mujumdar, R. B., Ernst, L. A., Mujumdar, S. R., Lewis, C. J., and Waggoner, A. S. (1993). Cyanine dye labeling reagents: sulfoindocyanine succinimidyl esters. Bioconjug. Chem. 4, 105-111. doi: 10.1021/bc00020a001

Murshudov, G. N., Skubák, P., Lebedev, A. A., Pannu, N. S., Steiner, R. A., Nicholls, R. A., et al. (2011). REFMAC5 for the refinement of macromolecular crystal structures. Acta Crystallogr. D Biol. Crystallogr. 67, 355-367. doi: $10.1107 /$ S0907444911001314

Ormö, M., Cubitt, A. B., Kallio, K., Gross, L. A., Tsien, R. Y., and Remington, S. J. (1996). Crystal structure of the Aequorea victoria green fluorescent protein. Science 273, 1392-1395. doi: 10.1126/science.273.5280.1392

Rizzo, M. A., Springer, G. H., Granada, B., and Piston, D. W. (2004). An improved cyan fluorescent protein variant useful for FRET. Nat. Biotechnol. 22, 445-449. doi: $10.1038 /$ nbt 945

Samma, A. A., Johnson, C. K., Song, S., Alvarez, S., and Zimmer, M. (2010). On the origin of fluorescence in bacteriophytochrome infrared fluorescent proteins. J. Phys. Chem. B 114, 15362-15369. doi: 10.1021/jp107119q

Sens, R., and Drexhage, K. H. (1981). Fluorescence quantum yield of oxazine and carbazine laser dyes. J. Lumin. 24-25, 709-712. doi: 10.1016/00222313(81)90075-2

Shaner, N. C., Campbell, R. E., Steinbach, P. A., Giepmans, B. N. G., Palmer, A. E., and Tsien, R. Y. (2004). Improved monomeric red, orange and yellow fluorescent proteins derived from Discosoma sp. red fluorescent protein. Nat. Biotechnol. 22, 1567-1572. doi: 10.1038/nbt1037

Shcherbakova, D. M., and Verkhusha, V. V. (2013). Near-infrared fluorescent proteins for multicolor in vivo imaging. Nat. Methods 10, 751-754. doi: 10.1038/nmeth. 2521

Shcherbo, D., Merzlyak, E. M., Chepurnykh, T. V., Fradkov, A. F., Ermakova, G. V., Solovieva, E. A., et al. (2007). Bright far-red fluorescent protein for whole-body imaging. Nat. Methods 4, 741-746. doi: 10.1038/nmeth1083

Shcherbo, D., Murphy, C. S., Ermakova, G. V., Solovieva, E. A., Chepurnykh, T. V., Shcheglov, A. S., et al. (2009). Far-red fluorescent tags for protein imaging in living tissues. Biochem. J. 418, 567-574. doi: 10.1042/BJ20081949

Shu, X., Royant, A., Lin, M. Z., Aguilera, T. A., Lev-Ram, V., Steinbach, P. A., et al. (2009). Mammalian expression of infrared fluorescent proteins engineered from a bacterial phytochrome. Science 324, 804-807. doi: $10.1126 /$ science. 1168683

Sineshchekov, V., Mailliet, J., Psakis, G., Feilke, K., Kopycki, J., Zeidler, M., et al. (2014). Tyrosine 263 in cyanobacterial phytochrome Cph1 optimizes photochemistry at the prelumi-R $\rightarrow$ lumi-R step. Photochem. Photobiol. 90, 786-795. doi: 10.1111/php. 12263

Song, C., Psakis, G., Lang, C., Mailliet, J., Gärtner, W., Hughes, J., et al. (2011). Two ground state isoforms and a chromophore D-ring photoflip triggering extensive intramolecular changes in a canonical phytochrome. Proc. Natl. Acad. Sci. U.S.A. 108, 3842-3847. doi: 10.1073/pnas.1013377108

Spillane, K. M., Dasgupta, J., Lagarias, J. C., and Mathies, R. A. (2009). Homogeneity of phytochrome $\mathrm{Cph} 1$ vibronic absorption revealed by resonance Raman intensity analysis. J. Am. Chem. Soc. 131, 13946-13948. doi: $10.1021 / j a 905822 \mathrm{~m}$

Spillane, K. M., Dasgupta, J., and Mathies, R. A. (2012). Conformational homogeneity and excited-state isomerization dynamics of the bilin chromophore in phytochrome Cph1 from resonance Raman intensities. Biophys. J. 102, 709-717. doi: 10.1016/j.bpj.2011.11.4019

Takala, H., Björling, A., Linna, M., Westenhoff, S., and Ihalainen, J. A. (2015). Light-induced changes in the dimerization interface of bacteriophytochromes. J. Biol. Chem. 290, 16383-16392. doi: 10.1074/jbc.m115.650127

Toh, K. C., Stojković, E. A., Rupenyan, A. B., van Stokkum, I. H., Salumbides, M., Groot, M. L., et al. (2011a). Primary reactions of bacteriophytochrome observed with ultrafast mid-infrared spectroscopy. J. Phys. Chem. A 115, 3778-3786. doi: 10.1021/jp106891x

Toh, K. C., Stojković, E. A., van Stokkum, I. H., Moffat, K., and Kennis, J. T. (2010). Proton-transfer and hydrogen-bond interactions determine fluorescence 
quantum yield and photochemical efficiency of bacteriophytochrome. Proc. Natl. Acad. Sci. U.S.A. 107, 9170-9175. doi: 10.1073/pnas.0911535107

Toh, K. C., Stojković, E. A., van Stokkum, I. H., Moffat, K., and Kennis, J. T. (2011b). Fluorescence quantum yield and photochemistry of bacteriophytochrome constructs. Phys. Chem. Chem. Phys. 13, 11985-11997. doi: $10.1039 / \mathrm{clcp} 00050 \mathrm{k}$

Wagner, J. R., Zhang, J., von Stetten, D., Günther, M., Murgida, D. H., Mroginski, M. A., et al. (2008). Mutational analysis of Deinococcus radiodurans bacteriophytochrome reveals key amino acids necessary for the photochromicity and proton exchange cycle of phytochromes. J. Biol. Chem. 283, 12212-12226. doi: 10.1074/jbc.M709355200

Weissleder, R. (2001). A clearer vision for in vivo imaging. Nat. Biotechnol. 19, 316-317. doi: 10.1038/86684

Yu, D., Gustafson, W. C., Han, C., Lafaye, C., Noirclerc-Savoye, M., Ge, W.P., et al. (2014). An improved monomeric infrared fluorescent protein for neuronal and tumour brain imaging. Nat. Commun. 5, 3626. doi: 10.1038/ ncomms 4626

Zepp, R. G., Sheldon, W. M., and Moran, M. A. (2004). Dissolved organic fluorophores in southeastern US coastal waters: correction method for eliminating Rayleigh and Raman scattering peaks in excitationemission matrices. Mar. Chem. 89, 15-36. doi: 10.1016/j.marchem.2004. 02.006
Zhang, J., Campbell, R. E., Ting, A. Y., and Tsien, R. Y. (2002). Creating new fluorescent probes for cell biology. Nat. Rev. Mol. Cell Biol. 3, 906-918. doi: $10.1038 / \mathrm{nrm} 976$

Zienicke, B., Chen, L. Y., Khawn, H., Hammam, M. A., Kinoshita, H., Reichert, J., et al. (2011). Fluorescence of phytochrome adducts with synthetic locked chromophores. J. Biol. Chem. 286, 1103-1113. doi: 10.1074/jbc.M110.155143

Zienicke, B., Molina, I., Glenz, R., Singer, P., Ehmer, D., Escobar, F. V., et al. (2013). Unusual spectral properties of bacteriophytochrome Agp2 result from a deprotonation of the chromophore in the red-absorbing form Pr. J. Biol. Chem. 288, 31738-31751. doi: 10.1074/jbc.M113.479535

Conflict of Interest Statement: The authors declare that the research was conducted in the absence of any commercial or financial relationships that could be construed as a potential conflict of interest.

Copyright $\odot 2015$ Lehtivuori, Bhattacharya, Angenent-Mari, Satyshur and Forest. This is an open-access article distributed under the terms of the Creative Commons Attribution License (CC BY). The use, distribution or reproduction in other forums is permitted, provided the original author(s) or licensor are credited and that the original publication in this journal is cited, in accordance with accepted academic practice. No use, distribution or reproduction is permitted which does not comply with these terms. 